\title{
Research on the Service of Subject Librarian in University Library under the new Media Environment
}

\author{
xiuli- $\mathrm{Li}^{1, \mathrm{a}^{*}}$ \\ ${ }^{1}$ Jilin business and technology college, ChangChun, JiLin, China \\ alixiulibb@126.com
}

Keywords: New media; University Library; Subject librarian; Subject service

Abstract. This paper investigates the subject librarian service of 211 Academic Libraries in 6 universities of Northeast region, and points out that in the new media era, the existing duties and services need to be changed in order to adapt to the new environment.

\section{新媒体环境下高校图书馆学科馆员的服务研究}

\author{
李秀郦 ${ }^{1, a *}$ \\ ${ }^{1}$ 吉林工商学院, 长春, 吉林, 中国 \\ alixiulibb@126.com
}

关键词: 新媒体 ; 高校图书馆; 学科馆员; 学科服务

中文摘要. 本文调查了东北地区6所211院校图书馆学科馆员服务情况, 指出在新媒体时代, 其现有的职责与服务急需改变, 以此来适应新环境下用户新的需求。

人类进行沟通传播的技术，从古至今从未间断过，其历史与人类社会的发展同步。从语 言到文字，从手抄到印刷，从电报到广播，从电脑到网络，媒介技术已经成为人类社会发展 的一个重要载体, 成为其不同历史时期的标志之一。以数字网络为基础的新媒体的诞生使人 类社会进入了一个空前快速发展的时期。新媒体已经彻底改变了我们的生活、学习的内容与 方式。本文通过对高校图书馆学科服务现状的调查, 探寻在新媒体时代, 读者新的需求, 我 们的图书馆，我们的服务应该达到一个怎样的标准，怎样带领读者领略新媒体的风采。

\section{1 新媒体的概念}

\section{1 技术说}

对于新媒体的定义，不同的专家学者可能会有不同的视角和观点，一般情况下，学者会 从技术特性的角度出发来定义。例如: 加州大学圣塔巴巴拉分校的教授 Ron Rice 在其著作 《新媒体：传播、研究与技术》中，指出 “那些可以使用户之间或者用户与信息之间进行互 动的一种传播技术, 通常是以电脑技术为基础。” ${ }^{[1]}$ 这是从技术层面上对新媒体定义比较有 代表性的界定。

\section{2 行为说}

除了技术说之外, 还有些学者从行为的角度来对新媒体进行界定, 这是从新媒体所引发 的传播行为和实践的层面出发来寻找新媒体的独特之处。例如: 加州大学欧文分校的教授波 
斯特曾指出 “新媒体技术能够带给用户前所未有的收获, 这样的一种收获能够对信息进行再 加工和再传播, 所以使新媒体相较传统媒体而言, 更难以控制。” ${ }^{[2]}$ 他认为, 新媒体的出现 使人们的行为发生变化，让很多以前不敢想的事情在新媒体时代成为现实。

\section{3 组织说}

社会组织及结构是成就新媒体成的一个重要因素。Kevin Robins 在《新媒体新在哪里》 给出一个定义 “新媒体创造了一个新的知识空间和传播空间，这一空间使人们脱离了原有的 线性的等级的和僵化的本土知识形态和关系，形成了一种离散的复杂的和灵活的知识体验。” ${ }^{[3]}$ 新媒体的诞生, 让全民一起来参与的信息和知识生产、组织及整合的过程成为了可能。

\section{4 三合一说}

不同的学者从不同的角度、不同的方向对新媒体定义进行了界定，可能这三个方面的定 义也不够诠释新媒体, 还有学者认为应该把这三种观点整合在一起, 即将媒介技术、人类行 为和社会结构整合起来。这样就打破了单纯从技术角度、行为角度或者组织角度对新媒体定 义的限定，基于以上三个层面，将新媒体的定义归纳为 “不同于传统大众媒体的包含特定技 术、实践和社会组织的信息与传播系统。” ${ }^{[4]}$ 这样就将技术层面的新媒体、行为层面的新媒 体和组织层面的新媒体整合起来，形成一种多范围新型的传播模式。

\section{2 东北地区高校图书馆学科服务现状分析}

本次主要是对东北地区的 6 所 211 院校开展的学科服务进行的调查, 采用问卷、网络及 访谈的方式进行, 调查对象主要为教师, 研究人员和在校研究生。调查共发放问卷 180 份, 回收 160 份, 有效问卷 160 份, 主要是围绕 6 所高校学科馆员的基本信息和服务内容展开的 调查, 以了解在新媒体时代, 各高校的学科服务真实情况, 期待为用户提供深层次的信息服 务。

\section{1 学科馆员数量}

调查结果显示, 学科馆员数量最多的高校为东北师范大学 ${ }^{[5]}$ 和东北大学 ${ }^{[6]}$, 都是 20 名, 其中东北师大有 7 名核心学科馆员, 13 名辅助学科馆员。其次是吉林大学 ${ }^{[7]}$, 学科馆员数量 为 11 名, 其中中心馆 3 名, 工学馆 2 名, 医学馆 2 名, 地学馆 3 名, 信息学馆 1 名。数量 最少的高校为哈尔滨工业大学 ${ }^{[8]}$, 目前只提供学科服务, 没有单独列出学科馆员名单。具体 详情见图 1。

\section{2 学科馆员素质}

调查结果显示，在 6 所高校中，学科馆员素质方面列的最全的是东北大学，在 20 名馆 员中, 有 7 名副研究馆员, 10 名馆员, 3 名助理馆员; 在东师图书馆 20 名馆员中, 大部分 都是副研究馆员或者硕士以上, 特别是在 7 名核心馆员中, 有 5 名博士学位, 另外 2 名为博 士在读; 吉林大学的学科馆员大部分为副高级职称, 而东北林业大学 ${ }^{[9]}$ 、哈尔滨工业大学和 大连海事大学则没有详细的介绍。

\section{3 学科馆员联系方式}

调查结果显示, 在学科馆员的联系方式上, 写的最全的学校为东北林业大学, 为电话、 QQ 和邮箱三种方式; 注一种联系方式的有三所学校, 分别为吉林大学的电话方式、哈工大 的学科博客方式和大连海事大学 ${ }^{[10]}$ 的邮箱方式, 其余都为邮箱和办公电话。

\section{4 涉及的学科}

调查结果显示, 在 6 所高校学科馆员服务中，服务最全面的是东北师范大学，共计 20 个学院, 60 多学科, 几乎全覆盖; 其次是东北大学, 服务涉及 3 个学院的 16 个学科; 大连 海事大学服务的是 4 个学院, 11 个学科, 目前吉林大学没有详细列出其服务学科。 


\section{5 学科馆员服务内容}

调查结果显示，到目前为止，这六所学校的服务内容都比较全面，例如，咨询服务，资 源建构, 开展培训, 资源推送等基础服务都在正常有序的进行着, 东北师大和东北大学开展 的内容最多, 形式也比较新颖, 例如科研分析、嵌入科研、嵌入教学及建设学科服务平台等, 哈工大还开设了学科博客服务, 具体服务内容见表 1 。

表 1 学科馆员服务内容分布图
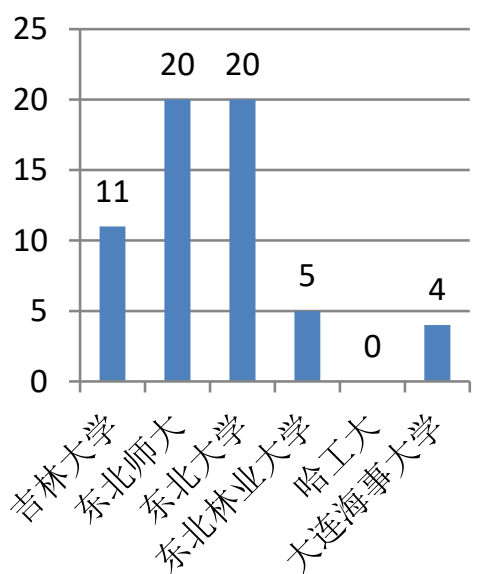

\begin{tabular}{|c|c|}
\hline 学校名称 & 服劳内容 \\
\hline 吉林大学 & $\begin{array}{c}\text { 选定图书、建立档案、开展培训、资源推 } \\
\text { 送、咨询服务、科研分析 }\end{array}$ \\
\hline $\begin{array}{l}\text { 东北师范 } \\
\text { 大学 }\end{array}$ & $\begin{array}{l}\text { 开展培训及检索课、科研分析、科技查新及查 } \\
\text { 收查引、嵌入教学科研、学科服务平台 }\end{array}$ \\
\hline 东北大学 & $\begin{array}{l}\text { 学科联络、嵌入教学、资源建设、开展培 } \\
\text { 训、2个学科服务平台、咨询服务 }\end{array}$ \\
\hline $\begin{array}{l}\text { 东北林业 } \\
\text { 大学 }\end{array}$ & $\begin{array}{c}\text { 调研沟通、资源建设、宣传培训、信息服 } \\
\text { 荌、咨询服务 }\end{array}$ \\
\hline $\begin{array}{l}\text { 哈尔滨工 } \\
\text { 业大学 }\end{array}$ & $\begin{array}{c}\text { 学科博客、经管、材料心理学、土木工程 } \\
4 \text { 个学科服务平台 } \\
\end{array}$ \\
\hline $\begin{array}{l}\text { 大连海事 } \\
\text { 大学 }\end{array}$ & $\begin{array}{c}\text { 资源荐构、㾔洵服务、开展培训、专题服 } \\
\text { 务 }\end{array}$ \\
\hline
\end{tabular}

图 1 学科馆员数量分布图

通过以上调研发现，当前高校图书馆开展的学科服务情况总体来说不是很好，主要表 现为对学科馆员的重视上, 有的学校没配备学科馆员, 有的学校配置的数量少; 在涉及服务 学科上, 没有详细列出具体学科, 使读者不能一目了然找到所需要的信息; 在馆员的素质方 面, 大部分高校没有详细列出其自身资质情况, 没有正规的标准; 在联系方式上, 目前还比 较单一的只有办公电话和邮箱, 很难做到国家倡导的 24 小时服务; 在嵌入教学科研、跟踪 服务方面, 深度也不够, 目前也都是被动的接受, 很多学校都没有主动提供服务, 虽然都已 经开展了学科服务, 但是服务效果不容乐观。

\section{3 新媒体环境下学科服务的策略}

\section{1 服务时间与空间}

学科服务的内容主要是网络资源的服务，因此应不受时间和空间的限制，可以随时随地 提供服务, 只要服务方式到位即可。在 2015 年 12 月 31 日, 教育部印发的《普通高等学校 图书馆规程》第六章第二十九条 “图书馆在学校教学时间内开馆每周应不低于 90 小时，假 期也应有必要的开放时间, 有条件的学校可以根据实际需要全天开放; 网上资源的服务应做 到全天 24 小时开放。” ${ }^{[11]}$ 明确指出了网上资源的服务应该是 24 小时。例如: 中国矿业大学 通过学科博客来开展 24 小时学科服务, 采用 “融入一线、嵌入过程, 用户在哪里, 服务就 在哪里” 的服务理念, 使得学科馆员或者说是图书馆与师生都达到了 “双赢” 的状态。这说 明在新媒体时代，学科服务超越时空界限来提升图书馆的服务水平成为了可能。

\section{2 服务内容}

调查发现，大部分馆里的学科服务内容还停留在咨询服务、培训服务、资源荐购、资料 的查询与检索等浅层面的服务, 很难满足用户深层次的需求。伴随着信息的快速发展, 用户 的需求也越来越深、越来越广, 自然, 馆员的服务也应越来越深、越来越广。例如: 在广度 上, 当前的学科服务对象还主要集中在学校的教师队伍上, 忽视了一个很重要的群体用户, 那就是研究生群体, 他们的学科服务需求比教师的还要强烈, 特别是论文撰写、课题查新、 
等需求尤为迫切, 所以学科服务应拓展用户服务范围。在深度上, 学科馆员要真正地嵌入教 学, 嵌入科研, 这是图书馆在新媒体时代发展服务的标志, 是提高其服务水平最有效的途径。

\section{3 服务方式}

新媒体时代，学科馆员的服务方式应该是多样性的。这是信息增长的速度和范围决定的， 仅仅使用办公电话和邮箱，这样单一的方式提供服务，至少在回复的时效性上是有缺陷的。 要想真正的开展学科服务并收到预期的效果，必须采用 “新媒体” 技术且是集多种方式综合 使用的。例如, 建立学科服务 $\mathrm{QQ}$ 交流群, 学科服务微信公众号、学科服务论坛、学科博客 及 RSS 信息订阅等多种交流方式，来发布学术热点、学科趋势及科研前沿等相关信息。

\section{4 学科馆员角色}

学科馆员是学科服务的主力、核心和灵魂, 主要负责学科资源的检索与整理, 学科平台 的管理与维护, 深入到教学、科研一线来提供服务等工作, 这就要求馆员的专业素质和综合 素质并重, 不仅要掌握传统文献的服务能力, 还要有时代信息技术的觉察力, 学习新技术、 新知识的主动意识, 掌握 “新媒体” 传播载体的技能。在提供服务时要对服务对象不同层次 用户的需求出发。因此, 新媒体时代, 学科馆员的角色是多样化的, 不仅是平台管理与维护 的技术员, 学生服务的辅导员, 还要成为嵌入教学科研的知识管理员, 多重身份的集合才能 更好地为全校师生提供精准有效的服务。

\section{4 结语}

新媒体时代的到来，学科服务是是高校图书馆实现服务价值的重要途径。她是一项集馆 员专业素质与综合素质为一体的服务, 要求馆员改变服务理念、更新知识结构, 要有创新性、 前瞻性、知识储备与共享意识, 学科馆员的素质高低直接决定了服务的质量, 同时也体现了 图书馆真实的服务水平，学科服务的发展程度标志着高校图书馆服务的深度和广度。在新媒 体时代, 在新媒体技术的推动下, 高校应积极重视并发展学科服务, 提升服务水平, 发挥图 书馆在学校的教学、科研工作中应有的作用, 让师生体会到图书馆无处不在的服务。

\section{致谢}

本文系吉林省教育厅 2016 年 “十三五”重点科研项目“论 MOOC 环境下高校图书馆服 务模式转变的研究” (项目编号: 吉教科文合字 [2016] 第 110 号) 研究成果之一; 吉林省 教育科学规划课题+慕课理念下高校信息检索课的理论设计与实践研究+GH16461 研究成果之

\section{References}

[1][2][3][4]http://mooc.guokr.com/note/15096/.2016-6-3.

[5]http://www.library.nenu.edu.cn/Menu/ServiceGuide/XGYY/XKFWJJ.aspx.2016-6-21.

[6]http://www.lib.neu.edu.cn/page/fw/xkfw/.2016-6-21.

[7]http://lib.jlu.edu.cn/portal/service/xuekefuwu.aspx.2016-6-9.

[8]http://www.lib.hit.edu.cn/subplat/xue-ke-fu-wu-ping-tai-0.2016-6-9.

[9]http://lib.nefu.edu.cn/fw/xkfw/xkgymd/.2016-6-9.

[10]http://lib.dlmu.edu.cn/dzfw/xkgyfw.html.2016-6-13.

[11] General library regulations. Ministry of Education of the People's Republic of China [Z].2015$12-31$. 